\section{Cureus}

\title{
Otogenic Brain Abscess: A Mini Review
}

\author{
Venkataramana Kandi ${ }^{1}$ \\ 1. Department of Microbiology, Prathima Institute of Medical Sciences
}

$\square$ Corresponding author: Venkataramana Kandi, ramana20021@gmail.com

Disclosures can be found in Additional Information at the end of the article

\section{Abstract}

Intracranial complications secondary to ear infections is an established clinical condition. The proximity of the brain compartments to the ear increases the chances of infections being percolated to central nervous system. Early clinical and laboratory diagnosis of such infections and better patient management strategies are required to minimize the intracranial and extracranial complications. Acute and chronic suppurative otitis media (CSOM) have been considered as risk factors for otogenic brain complications. Among the microbiological causes, recently there has been an increase in the cases of otogenic brain abscess due to Streptococcus spp. This review discusses the microbiological causes, implications, laboratory, diagnosis, and management of otogenic complications of central nervous system.

Categories: Neurology, Infectious Disease

Keywords: otogenic brain abscess, streptococcus spp, intracranial complications of middle ear infections

\section{Introduction And Background}

Brain abscess is defined as localized inflammation and infection of the brain parenchyma. Although uncommon, brain abscess remains to be a serious and life threatening clinical condition that warrants immediate medical attention to reduce the resultant morbidity and mortality [1]. Previous studies have noted that there is no age predilection, but more than a quarter of brain abscesses are seen in children and old-age groups [2]. Over the years, it was observed that incidences of otogenic brain infections have come down due to increased use of antimicrobial agents [3]. The availability of non-invasive laboratory techniques for a better understanding of the involvement of brain parenchyma including the computed tomography (CT scan) and magnetic resonance imaging (MRI) of brain have been instrumental in better patient care and treatment outcome [4]. Aetiology of brain abscess is complex, which may involve a single microbe (bacteria, fungi, parasite), polymicrobial, and involvement of anaerobic bacteria. Otogenic brain infections have been noted both in immunocompetent and immunocompromised individuals. The predisposing factors for brain abscess include repeated upper respiratory tract infections, sinusitis, chronic suppurative otitis media, trauma, solid organ transplant recipients, acquired immunodeficiency syndrome (AIDS) patients, dental manipulations/infections, and post-neurosurgical procedures. Depending on the primary infection site, the type of central nervous system (CNS) involvement can be understood. Ethmoidal and sphenoid sinusitis results in infection of frontal lobe and temporal lobe infection of brain, respectively. Infection of the middle ear and mastoids may spread to CNS involving mostly the cerebellum and temporal lobes. Hematogenous spread of infection from primary sites to CNS like the dental infections, lung abscess, and other solid organ infections have been reported in literature [5]. Brain abscess involves four stages where, in the first stage, the bacteria invades in to the brain tissue resulting in neutrophil, astrocytes and microglial cell activation, edema, and tissue necrosis. The second stage involves spread of the infection to 
adjacent brain tissue and further invasion of macrophages, lymphocytes, and resultant necrosis. The third stage is characterized by the development of a vascularised capsule surrounding the lesion that appears on CT scan as a ring-enhancing lesion. The final stage of a brain infection results in immune response resulting in the destruction of capsule and further involvement of surrounding healthy brain parenchymal cells. The pathogenesis of brain infection depends on the immune status of the patient, type/virulence of microbe involved, and the use of antimicrobial agents [5].

\section{Review}

\section{Microbiology of brain abscess}

Brain abscess resulting from a contagious site of infection (ear infection, sinusitis) may most often involve the colonized bacteria of the oral and upper respiratory tract. Anaerobic bacterial flora forms a significant part of the colonized bacteria in the oral cavity (Fusobacterium spp, Bacteroides spp, Prevotella spp, and the anaerobic Streptococci). Staphylococcus aureus, coagulase-negative Staphylococci, Pseudomonas spp, enteric bacteria, Streptococcus spp, and Clostridium spp are more common among the patients with sinusitis and trauma.

Hematogenous spread of bacteria to brain involving viridians Streptococci, Haemophilus, and Enterococci may happen in patients with congenital heart disease. Fungal (Cryptococcus spp, Aspergillus spp, Zygomycetes members, Histoplasma capsulatum, Blastomyces dermatitidis) and parasitic (Entamoeba histolytica, Angiostrongylus spp, Tinea spp, and others) cause of brain abscess is not uncommon and is most often seen in immunocompromised and debilitated individuals [6-10].

\section{Clinical manifestation of brain abscess}

Clinical symptoms during a brain abscess depend on the type and location of the lesion, extent of infection, type of microorganism involved, and the immune status of the patient. Headache is the most common complaint during a brain abscess, and patients may present with/without fever, nausea, vomiting, altered mental status, and lethargy. Patients may also present with focal neurological deficits like hemiparesis, motor speech deficit, aphasia, papilledema, and visual field defects. Spread of the infection to the meninges may result in symptoms like the neck stiffness and seizures. Involvement of the cerebellum is observed as a most common consequence in otogenic brain abscess; the clinical symptoms seen include ataxia, dysmetria, and nystagmus $[1,5]$.

\section{Laboratory diagnosis of brain abscess}

Laboratory diagnosis of brain abscess involves both invasive and non-invasive procedures. Non-invasive imaging techniques include computed tomography (CT scan) and magnetic resonance imaging (MRI) of the brain, which may be very beneficial in studying the extent of infection and also useful for prognosis. Microbiological evaluation includes the blood culture that confirms hematogenous spread of infection. A lumbar puncture can be done to collect cerebrospinal fluid (CSF) for microbiological and cellular analysis. Tests for antigen detection of Cryptococcus spp, serological evidence of antibodies against Toxoplasma, and C-reactive protein (CRP) estimation to evaluate the inflammatory condition can also be performed based on their availability. CT-guided aspiration of abscess pus is the most common procedure performed and the pus is evaluated microbiologically. Gram's stain, acid-fast stain, aerobic, and anaerobic cultures are performed to confirm/rule out infection with bacteria and Mycobacterium tuberculosis. When the patient is immunocompromised, additional staining and culture methods are employed for isolation of rare microbial species like the Nocardia spp, Rhodococcus spp, and others. Cytopathologic and histopathologic studies of the affected brain tissue may be useful to rule out malignancy [11-13]. 


\section{Management of otogenic brain abscess}

Otogenic brain abscess has been a cause for severe morbidity and mortality in the developing world. The cost associated with the diagnosis, treatment, and management of the intracranial complications of otogenic brain abscess should be considered as a cause of serious concern.

Clinical diagnosis of brain abscess followed by radiological confirmation is necessary to initiate treatment and for assessing the prognosis. Conventionally, the management of otogenic brain abscess involves aspiration of the pus from the abscess, microbiological, cytological, and histopathological examination. Appropriate antimicrobial chemotherapy is initiated, followed by a decision on the need to surgical intervention is taken. Depending on the extent of involvement of the brain compartments, the size, and numbers of abscesses, neurosurgical drainage is performed either through open craniotomy and drainage or by drainage through a burr hole. Post-drainage, mastoidectomy can be done to avoid recurrence and remove the source of infection. Identification of microbial cause and antimicrobial susceptibility profile guided specific intravenous antibiotic treatment initiation was found to show better patient prognosis [14-16].

\section{Case study}

A 29-year-old male patient presented to the emergency department with symptoms of nausea, vomiting, severe headache, giddiness, difficulty in ingesting food, and difficulty in walking. The patient gave a history of ear discharge since one month. The patient was apparently healthy one month back. The ear discharge was from the left ear and was whitish in colour and foul smelling. The patient revealed that he had on and off ear infections since childhood. The patient was a non-smoker and used alcohol occasionally. There was no history of diabetes mellitus, hypertension, asthma, or tuberculosis. He was non-reactive for human immunodeficiency virus (HIV), hepatitis B virus (HBV), and hepatitis C virus (HCV). A CT scan, $5 \mathrm{~mm}$ slice thick, was taken in 128 slices without intravenous catheterization. The CT scan of the brain revealed evidence of a hypodense lesion with surrounding perilesional oedema in left cerebellar region. The CT scan also showed evidence of erosion of the sigmoid plate with extension of collection of middle ear into the intracranial cavity.

A MRI was performed for a better study of the brain, which confirmed left CSOM with extension into the left cerebellum with perilesional oedema causing compression of pons, fourth ventricle aqueduct, and minimal middle line shift of the right side. The MRI also showed evidence of a T1 hypointense and T2 hyperintense lesion in the left mastoidal cells, left external ear, and middle ear cavity with extension to lateral part of the left cerebellum. Perilesional oedema surrounding left cerebellum and vermis, causing mass effect in the form of compression of the fourth ventricle aqueduct and pons with a minimal midline shift towards right side, was confirmed. The remainder of the brain tissue was found normal. Based on the clinical symptoms and results of the imaging studies, a diagnosis of otogenic cerebellar abscess was made. Computer-guided aspiration of the abscess pus was performed and was sent for microbiological analysis (Figure 1). Gram's stain of the pus showed occasional gram-positive cocci in singles and pairs (Figure 2). Acid-fast staining for tubercule bacilli was negative. The aspirated pus showed no growth of anaerobic bacteria. Culture for fungus was negative. Aerobic culture revealed growth of pinpoint semi-translucent non-hemolytic bacteria on blood agar, which on gram staining are confirmed as gram-positive cocci in pairs and chains. 


\section{Cureus}

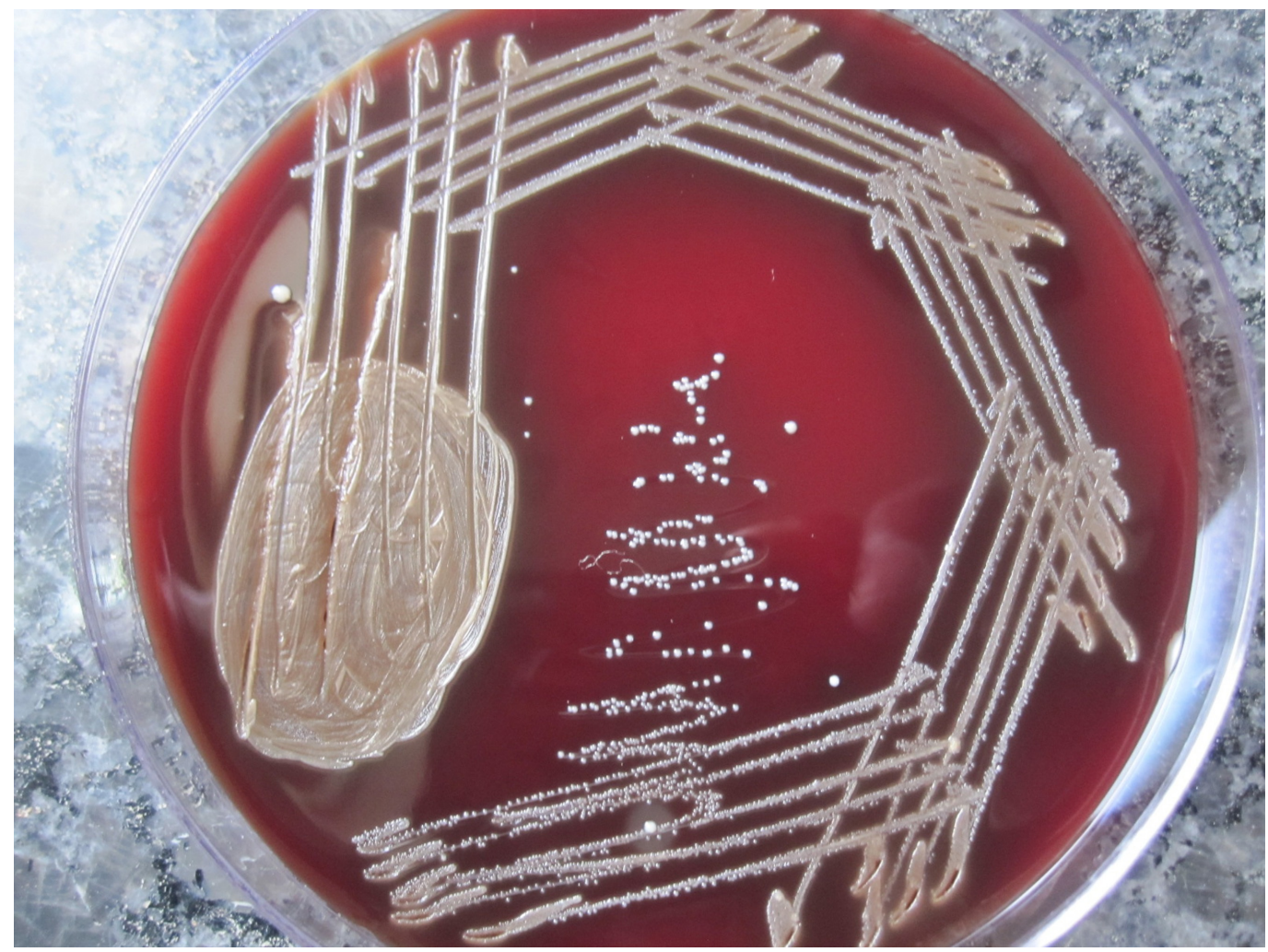

FIGURE 1: Growth of Streptococcus spp on blood agar

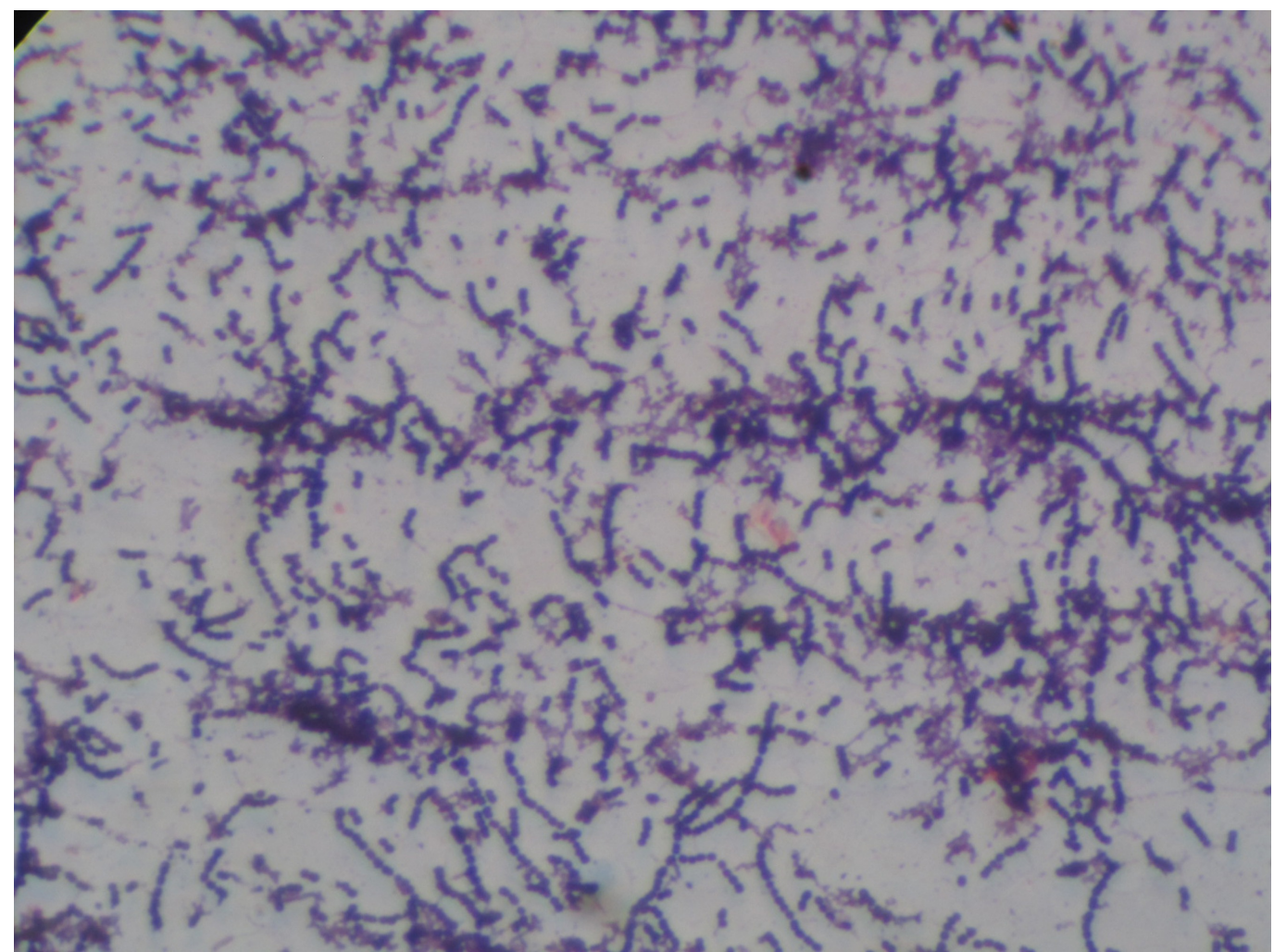

FIGURE 2: Gram's stain picture of Streptococci appearing in pairs and chains 


\section{Cureus}

There was no growth on MacConkey's agar. The bacteria were non-sporing, non-motile, oxidase-negative, catalase-negative, and non-acid fast. Based on the biochemical and cultural characters, the bacterium was identified as Streptococcus spp. The antimicrobial susceptibility testing of the bacteria revealed sensitivity to linazolid, gentamicin, ofloxacillin, clindamycin, co-trimoxazole, amoxycillin-clavulinic acid, azithromycin, and imipenem (Figure 3). The isolated bacterium was resistant to cetftazidime, cefaperazone, and ceftriaxone.

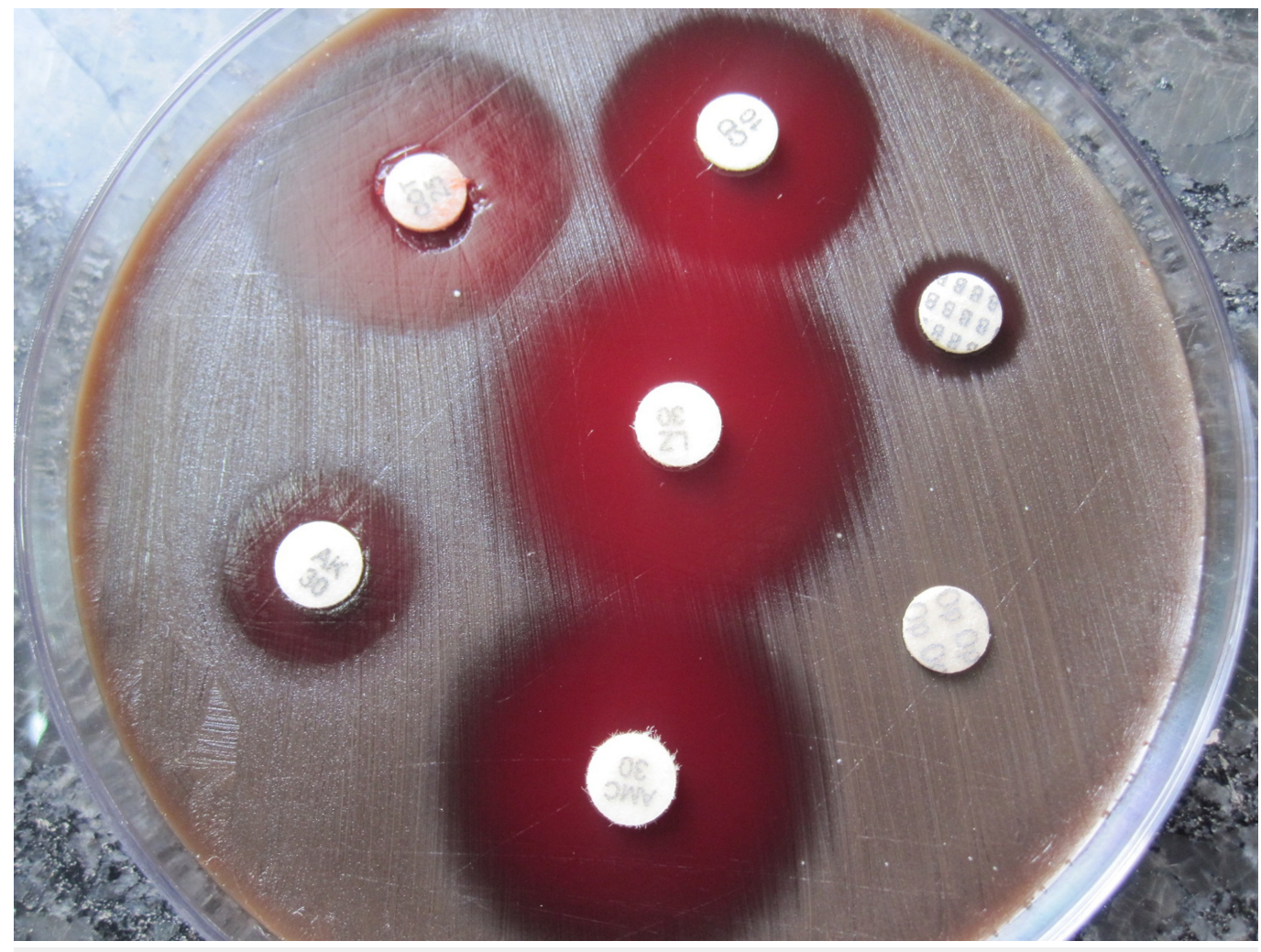

\section{FIGURE 3: Antimicrobial susceptibility testing of Streptococci on blood agar}

Due to the unavailability of antisera, the Streptococcus isolated was not identified to the species level.

Otogenic brain abscess is defined as accumulation of pus in the cerebellum as a result of recurrent middle ear infection. Appropriate clinical and laboratory diagnosis and prompt initiation of antimicrobial chemotherapy is essential to reduce the resultant morbidity and mortality. Mortality of the brain abscess cases is found to be more than $25 \%$ and, if the patients are not properly managed, may lead to morbidity in $70 \%$ of the patients [17]. Otogenic CNS infections have been reported both in acute and chronic cases of otitis media [18]. In a study reported by Penido Nde, et al., a 15 year observation revealed that Proteus spp, Pseudomonas spp, Streptococcus spp, and Haemophilus spp were commonly associated with intracranial complications secondary to otitis media [19]. Another study has reported that chronic otitis media is a risk factor for both intracranial and extracranial complications [20]. A retrospective study by Dubey, et al. among patients suffering from chronic middle ear infection showed that $40.6 \%$ of patients were aged over 18 years and more than $30 \%$ of the patients were between $0-10$ years. The same study has revealed that among the CNS involvement, 56.3\% had single lesion 
intracranial complications and $43.7 \%$ had multiple intracranial complications. Meningitis was the most common intracranial complication (43.7\%), followed by lateral sinus thrombosis (31.2\%), cerebellar abscess (18.7\%), epidural abscess (21.8\%), perisinus abscess

(15.6\%), cerebral abscess and interhemispheric abscess (6.2\%), and subdural abscess, otitic hydrocephalus, and otogenic cavernous sinus thrombosis patients (3.1\%) [21]. Recently, the most common microbiological cause for otogenic cerebellar abscess was found to be Streptococcus spp, including the Enterococci as reported in the literature [22-26]. A recent study from India has reported that among the 57 cases of brain abscess, Streptococcus spp (36.8\%) was the most common causative, followed by Staphylococcus aureus (22.8\%) and Pseudomonas spp (5.2\%) [27]. Streptococci are a group of facultatively anaerobic gram-positive cocci arranged in pairs and chains belonging to the family, Streptococcaceae. Members of Streptococci are present as normal flora in humans and animals and are recognized as potential pathogens in humans. Laboratory identification of Streptococci depends mainly on the type of the haemolysis they produce in sheep blood agar and the Lancefield serogrouping (A-V, except I and J). Streptococci have been most commonly associated with sore throat, pharyngitis, and tonsillitis (Group A Streptococcus/Streptococcus pyogenes). Skin infections (pyoderma- abscess, erysipelas, impetigo, cellulitis, etc.), urinary tract infections, wound infections, endocarditis, pneumonia, polyarthritis, septicaemia, and meningitis are other infections caused by various Streptococcus spp. Predisposing factors for infections with Streptococci include cardiovascular diseases, diabetes mellitus, liver and kidney diseases, and chronic infections [28]. As many laboratories are ill-equipped to isolate anaerobic microorganisms, and since brain abscess may involve both aerobic and anaerobic microorganisms, it is suggested that antimicrobial chemotherapy should also include antibiotics against anaerobic organisms [29-31].

\section{Conclusions}

Brain abscess is a serious clinical condition, which, if not timely diagnosed, may result in severe morbidity and mortality. Identification of predisposing factors and effective patient management strategies are essential for better prognosis. Most cases of brain abscess have been noted in patients with the primary infection of a different origin. Although haematogenous spread of infection to the brain is not uncommon, the frequent cause of intracranial invasion of microorganisms is from the ear infections. Therefore, physicians, otologists, clinical microbiologists, and neurospecialists should consider all factors for the better management of patients.

\section{Additional Information}

\section{Disclosures}

Conflicts of interest: In compliance with the ICMJE uniform disclosure form, all authors declare the following: Payment/services info: All authors have declared that no financial support was received from any organization for the submitted work. Financial relationships: All authors have declared that they have no financial relationships at present or within the previous three years with any organizations that might have an interest in the submitted work. Other relationships: All authors have declared that there are no other relationships or activities that could appear to have influenced the submitted work.

\section{Acknowledgements}

I acknowledge the support of the management, Prathima Institute of Medical Sciences, Karimnagar, India. I also Thank the Department of Radiology for providing the radiographs.

\section{References}

1. Lu CH, Chang WN, Lin YC, Tsai NW, Liliang PC, Su TM, Rau CS, Tsai YD, Liang CL, Chang CJ, 
Lee PY, Chang HW, Wu JJ: Bacterial brain abscess: Microbiological features, epidemiological trends and therapeutic outcomes. QJM. 2002, 95:501-9.

2. Bartnik W, Bartnik-Krystalska A: Results of the therapy in otogenic intracranial complication in ENT Department of Voivodeship Hospital in Kalisz (article in Polish). Otolaryngol Pol. 2000, 54:383-8.

3. Hafidh MA, Keogh I, Walsh RM, Walsh M, Rawluk D: Otogenic intracranial complications. A 7-year retrospective review. Am J Otolaryngol. 2006, 27:390-5.

4. Rosenblum ML, Hoff JT, Norman D, Weinstein PR, Pitts L: Decreased mortality from brain abscesses since advent of computerized tomography. J Neurosurg. 1978, 49:658-68.

5. Tonon E, Scotton PG, Gallucci M, Vaglia A: Brain abscess: Clinical aspects of 100 patients. Int J Infect Dis. 2006, 10:103-9.

6. Ionita C, Wasay M, Balos L, Bakshi R: MR imaging in toxoplasmosis encephalitis after bone marrow transplantation: Paucity of enhancement despite fulminant disease. AJNR Am J Neuroradiol. 2004, 25:270-3.

7. Kielian T, Haney A, Mayes PM, Garg S, Esen N: Toll-like receptor 2 modulates the proinflammatory milieu in Staphylococcus aureus-induced brain abscess. Infect Immun. 2005, 73:7428-35.

8. Grigoriadis E, Gold WL: Pyogenic brain abscess caused by Streptococcus pneumoniae: Case report and review. Clin Infect Dis. 1997, 25:1108-12.

9. Obana WG, Scannell KA, Jacobs R, Greco C, Rosenblum ML: A case of Rhodococcus equi brain abscess. Surg Neurol. 1991, 35:321-4.

10. Farrar DJ, Flanigan TP, Gordon NM, Gold RL, Rich JD: Tuberculous brain abscess in a patient with HIV infection: Case report and review. Am J Med. 1997, 102:297-301.

11. Whelan MA, Hilal SK: Computed tomography as a guide in the diagnosis and follow-up of brain abscesses. Radiology. 1980, 135:663-71.

12. Liston TE, Tomasovic JJ, Stevens EA: Early diagnosis and management of cerebritis in a child . Pediatrics. 1980, 65:484-6.

13. Sharma BS, Gupta SK, Khosla VK: Current concepts in the management of pyogenic brain abscess. Neurol India. 2000, 48:105-11.

14. Viswanatha B, Nsaeeruddin K: Conservative management of otogenic brain abscess with surgical management of attico antral ear disease: A review. Indian J Otolaryngol Head Neck Surg. 2012, 64:113-9. 10.1007/s12070-012-0524-7

15. Sennaroglu L, Sozeri B: Otogenic brain abscess: Review of 41 cases . Otolaryngol Head Neck Surg. 2000, 123:751-5.

16. Viswanatha B, Sarojamma, Vijayashree MS, Sumatha D: Unilateral attico antral ear disease with bilateral intracranial complications. Indian J Otolaryngol Head Neck Surg. 2012, 64:82-6. 10.1007/s12070-011-0127-8

17. Osma U, Cureoglu S, Hosoglu S: The complications of chronic otitis media: Report of 93 cases . J Laryngol Otol. 2000, 114:97-100.

18. Chew YK, Cheong JP, Khir A, Brito-Mutunayagam S, Prepageran N: Complications of chronic suppurative otitis media: A left otogenic brain abscess and a right mastoid fistula. Ear Nose Throat J. 2012, 91:428.

19. Penido Nde O, Borin A, Iha LC, Suguri VM, Onishi E, Fukuda Y, Cruz OL: Intracranial complications of otitis media: 15 years of experience in 33 patients. Otolaryngol Head Neck Surg. 2005, 132:37-42.

20. Dankuc D, Milosević D, Savić L: Simultaneous extracranial and intracranial otogenic complications (article in Croatian). Med Pregl. 2000, 53:409-12.

21. Dubey SP, Larawin V, Molumi CP: Intracranial spread of chronic middle ear suppuration . Am J Otolaryngol. 2010, 31:73-7. 10.1016/j.amjoto.2008.10.001

22. Pehlivan Y, Toy MA, Karaoglan I, Namiduru M, Buyukhatipoglu H: Enterococcus avium cerebral abscess. Intern Med. 2007, 46:1280.

23. Walsh B, Williams A E, Satsangi J: Streptococcus suis type 2: Pathogenesis and clinical disease. Rev Med Microbiol. 1992, 3:65-71.

24. Latha R, Rajbhaskar R, Kavitha K, Senthil Pragash D, Vinod R: Otogenous Temporal Lobe Brain Abscess Which was Caused by Enterococcus Faecalis: A Case Report. Journal of Clinical and Diagnostic Research. 2012, 6:902-904. www.jcdr.in/articles/PDF/2204/42\%20\%204186.\%20F.pdf.

25. Cho SH, Park MK, Lee JD, Hwang SC: Otogenic brain abscess presenting with gait ataxia . 


\section{Cureus}

Korean J Audiol. 2012, 16:31-34. 10.7874/kja.2012.16.1.31

26. Lim RSM, Baxter M, Tan KF, Haney M, Suen K: Otogenic Intracranial Abscesses: A Case Series. Journal of Case Reports. 2013, 3:413-418. http://www.casereports.in/articles/3/2/OtogenicIntracranial-Abscesses-A-Case-Series.html.

27. Sharma S, Malhotra S, Bhatia NJK, Wilson A, Hans C: Right temporal otogenic brain abscess by Enterococcus faecalis - A rare case report. Int J Curr Microbiol App Sci. 2014, 3:101-4. http://www.ijcmas.com/vol-3-3/S.Sharma,\%20et\%20al.pdf.

28. Center for Food Security and Public Health . (2014). Accessed: September 27, 2014: http://www.cfsph.iastate.edu.

29. Ingham HR, Selkon JB: Metronidazole and brain abscess. Lancet. 1982, 2:613-4.

30. Warner JF, Perkins RL, Cordero L: Metronidazole therapy of anaerobic bacteremia, meningitis, and brain abscess. Arch Intern Med. 1979, 139:167-9.

31. Skoutelis AT, Gogos CA, Maraziotis TE, Bassaris HP: Management of brain abscesses with sequential intravenous/oral antibiotic therapy. Eur J Clin Microbiol Infect Dis. 2000, 19:332-5. 